\title{
SSK Modulation with Single Photon Avalanche Diode
}

\author{
Song $\operatorname{Han}^{1, a}$, Jian Zhang ${ }^{1, b}$ and Jie Song ${ }^{2, c}$ \\ ${ }^{1}$ Information Engineering University, Zhengzhou 450001, China; \\ ${ }^{2}$ Haerbin Engineering University, Zhengzhou 450001, China. \\ a2909312638@qq.com, bswordrawn@163.com, 602218644@qq.com
}

Keywords: visible light communication; single photon avalanche diode; Space Keying Shift modulation.

\begin{abstract}
In this paper, a Space Keying Shift(SSK) system based on a Single Photon Avalanche Diode (SPAD) receiver is presented. SSK modulation has the advantage of faster transmission rate than OOK modulation, and the implementation complexity is not high, it will become a widely used modulation method in single photon detection. In this paper, the SSK system based on the low light environment of the darkroom is proposed, and the BER performance of the SSK modulation system is analyzed. The simulation results are in accordance with the theoretical derivation.
\end{abstract}

\section{Introduction}

With the continuous development of wireless communication services, the limited spectrum resource becomes the bottleneck the development of wireless communication, visible light communication (VLC) developed in recent years using visible light spectrum (380nm-780nm) new resources, provide a supplementary means for electromagnetic wireless communication, it is based on green lighting source from Light-Emitting Diode (LED), in order to solve the problem of "Spectrum Tension" , "Depth Coverage" and "Green Energy Saving" in wireless local area network provides a new means of communication, compared to the traditional way, visible light communication has the advantages of high transmitting power, no electromagnetic interference and energy saving[1,2]. Visible light communication system using intensity modulation / direct detection, the electric signal is first loaded on the power intensity of the visible light by a light-emitting diode (LED), and then by the receiving end of the photo diode(PD) or Avalanche Photo Diode(APD) to achieve photoelectric conversion[3,4]. However, the mode of this receiver is not efficient, the reason is that the sensitivity of the PD or APD is not enough, to enable the receiver to output a large amount of processable current, a trans-impedance is provided to provide amplification. However, in the long-distance transmission, the visible light signal received relatively weak, will be brought about by the trans-impedance of thermal noise, and ultimately limit the transmission distance of the visible light system [5]. Therefore, in order to solve the problem of limited distance of the visible light communication system, the latest solution is to adopt the single photon counting method to respond to the optical signal and introduce the multi-input and multi-output technology into the single photon detection visible light system at the same time. Single photon detector for visible light communication is Single Photon Avalanche Diodes, when there is a photon when the tube immediately lead to avalanche effect, the tube output high current as a count. This method not only utilizes the sensitivity of avalanche effect, but also protects the information loaded on the optical power from the avalanche effect by recording the number of photons. In this paper, the modeling, theoretical derivation and simulation of the single photon visible light communication system are carried out according to the existing darkroom model in the laboratory. In this paper, the basic OOK modulation scheme is introduced and the multi - input multiple - output (MIMO) based on single photon detection is studied. Space Keying Shift is the most basic MIMO method, only one transmitter unit works at any time, and the other transmit antennas are idle [6,7]. The feasibility of introducing MIMO into single-photon detection visible-light system is verified by SSK modulation. 
System Model. As shown in Figure 1, the communication system is a $5 \times 5 \times 3$ darkroom, LED light source is located on the ceiling of 3 meters high, the light level is 0.85 meters high desktop, the distance between the two lights $0.4 \mathrm{~m}, 0.1 \mathrm{~m}$ between the two SPAD.

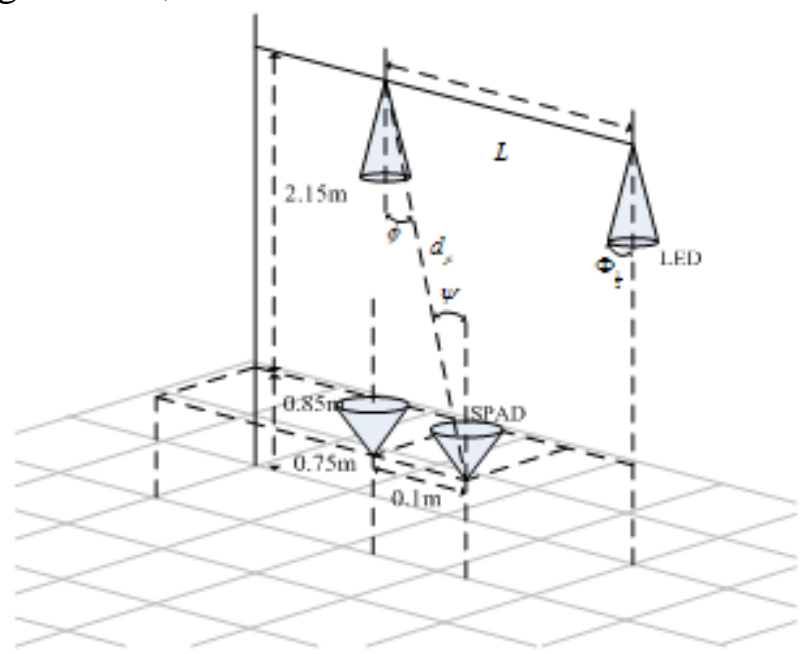

Fig. 1 SSK modulation system model diagram based on single photon detection

The dark room without background light and electromagnetic interference, SPAD detected light can only be emitted by the LED light. The light emission end meets the Lambertian radiation model:

$$
R_{0}(\phi)=\frac{(\beta+1)}{2 \pi} \cos ^{\beta}(\phi) P_{t}
$$

where $\beta=-\ln 2 / \ln \left(\cos \left(\Phi_{1 / 2}\right)\right)$, and $\Phi_{1 / 2}$ is the transmitter semi-angle which represents the half power angle. The variable $\phi$ denotes the angle of radiation, and $P_{t}$ is the average power of the LED. SPAD visible light communication using photon counting method to record signals, signal time slot in the optical power of the decision to record the number of photons, so the receiving end of the decision is based on the number of received photons. However, the randomness of the photon counting process will affect the accuracy of the decision, often this randomness as an additive noise, mathematical theory shows that the photon counting process obeys the Poisson distribution, so the final signal received by the SPAD Which is quite different from the Gaussian noise considered in the traditional PD detection method. SSK modulation as the most basic spatial modulation method, each time there is only one transmitter unit to work, other transmit antennas are idle. At the receiving end, SSK modulation detection equipment needs to first determine the transmission antenna used in the transmission of the index number, through the constellation to find the corresponding information bits, and then decoding operation to get the original send information. The SPAD-based SSK modem receiver is determined by the number of photons sent to the sender of the information, so we calculate the number of photons by the following formula:

$$
N_{p}=C_{P D E} \frac{(\beta+1) A p_{t}}{2 \pi d_{i j} E_{p}} \cos \phi
$$

Where $C_{P D E}$ is the value of the photon detection efficiency; A is the area of the SPAD receiver; and $d_{i j}$ is the distance between the different lamps to the different receivers; $E_{p}$ is the energy of a photon; Because of different transmitter and receiver position and lead to their different distances between the distance $d$ and the radiation angle $\varphi$ at the transmitter is different, so different receivers to receive two LED photon number will be different. 


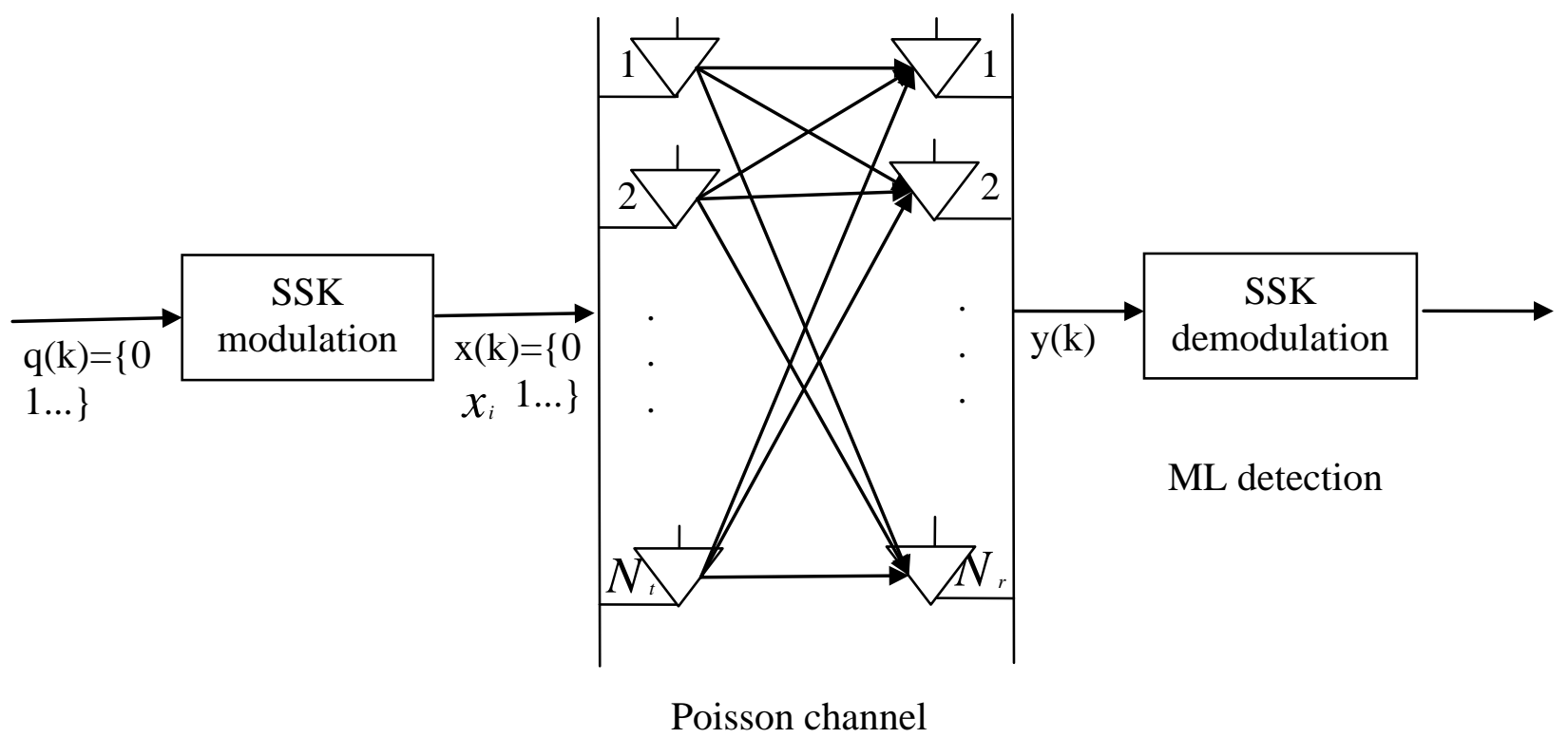

Fig. 2 SSK modulation system theory diagram based on single photon detection

As shown in the figure, the input bit is a sequence of $q(\mathrm{k})=\{0101 \ldots\}$. After mapping, 0 and 1 correspond to 01 and 10 matrix respectively. The matrix is the matrix of received photon number, then the photon number matrix is transformed into the output bits by the maximum likelihood detection to recover the information of the transmitting end.

\section{Simulation results}

Table 1 Simulation parameters

\begin{tabular}{cc}
\hline Experimental parameters & Value \\
\hline The PDE of the SPAD $C_{P D E}$ & $20 \%$ \\
The DCR of the SPAD $N_{D C R}$ & $7.27 \mathrm{kHz}$ \\
The energy of the photon $E_{P}$ & $4.42 \times 10^{-19} \mathrm{~J}$ \\
The hight of the room & $2.15 \mathrm{~m}$ \\
The size of the SPAD A & $5.04 \mathrm{~mm}^{2}$ \\
\hline
\end{tabular}

Under the condition that the above parameters are fixed, the BER performance of SSK modulation system with different transmission rate and half angle is simulated and compared, and the parameter values are optimized by changing the parameters. 


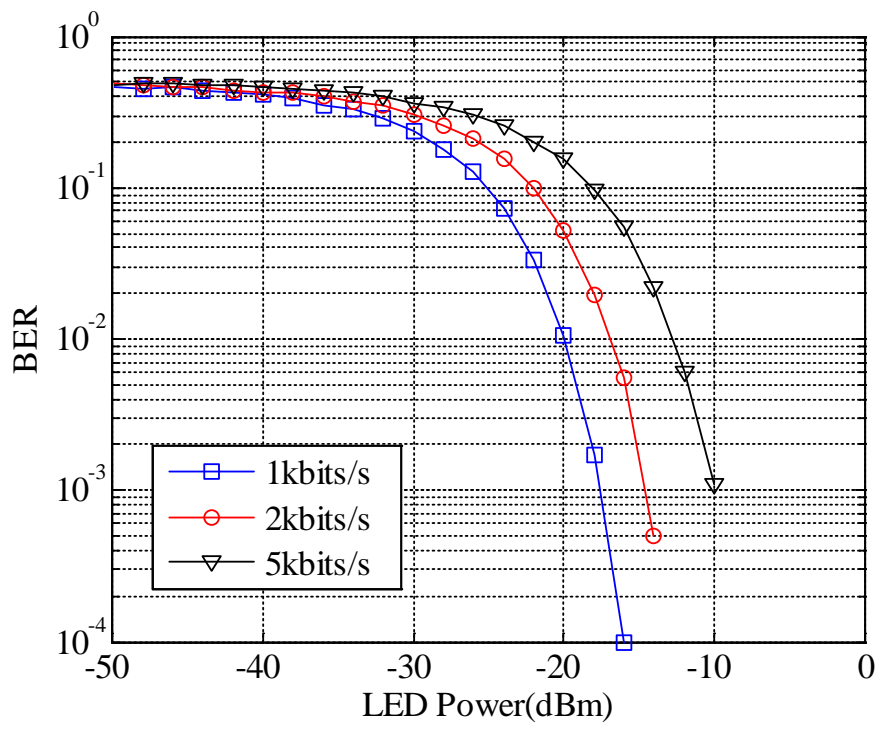

Figure 3 Under different rate of bit error rate performance of the SSK modulation system

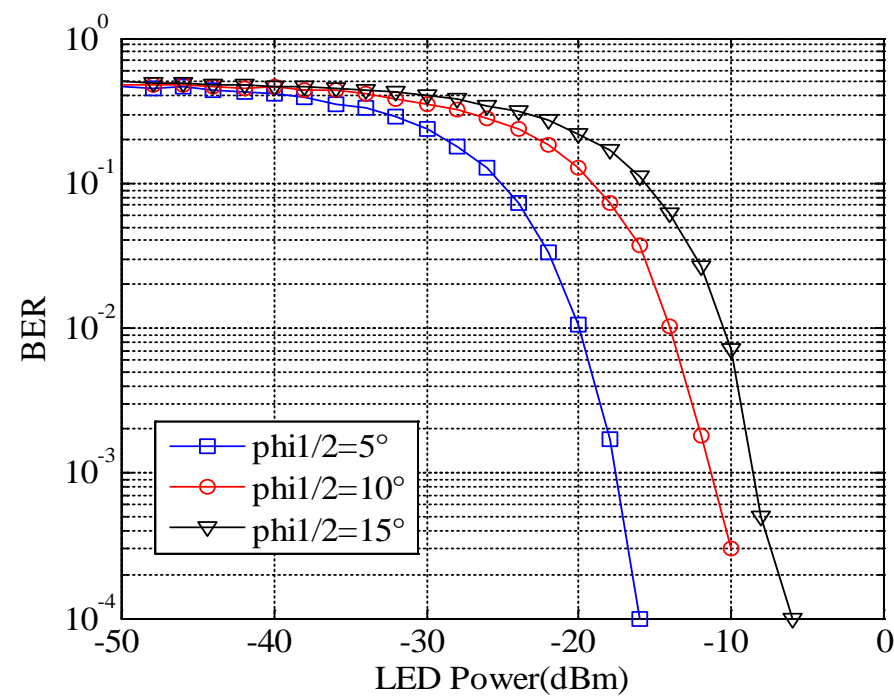

Figure 4 Under different optical power half angle of bit error rate performance of the SSK modulation system

Figure 3 shows the LED optical power half-angle of $10^{\circ}$, SPAD receiver bit error rate performance, the transmission rate is set to $1 \mathrm{kbits} / \mathrm{s}$, $2 \mathrm{kbits} / \mathrm{s}$ and $5 \mathrm{kbits} / \mathrm{s}$, can be seen with the transmission rate increases, The error performance is degraded. Figure 4 shows the fixed transmission rate of $1 \mathrm{kbits} / \mathrm{s}$, LED lights at different optical power half-angle of the whole system under the BER performance, we can see that with the increase of optical power half-angle, BER performance is reduced.

\section{Summary}

In this paper, a single-photon avalanche diode SPAD for a visible-light system based on single-photon detection technology and a receiver is described. The principle model of SSK modulation based on single photon detection is described and simulated in darkroom environment. The simulation results are in good agreement with the theoretical results, and the feasibility of SSK modulation based on single photon detection is verified.

\section{References}

[1] A. Jovicic, J. Li. and Tom Richardson, Visible Light Communication: Opportunities, Challenges and the Path to Market, IEEE Commun.Magaz.2013, 51(12):26-32. 
[2] S. K. Routray. The Changing Trends of Optical Communication, IEEE Potentials, 2014, 33(1): 28-33.

[3] N.Kumar and N.R.Lourenco, "Led-based visible light communication system: a brief survey and investigation,” J. Eng. Appl. Sci, vol.5, no. 4, pp. 296-307, 2010.

[4] P. H. Panthak, X. Feng, P. Hu, and P. Mohaparta, "Visible light communication, networking, and sensing: A survey, potential and challenge,” Communications Survey \& Tutorials, IEEE, vol. 17, no. 4, pp. 2047-2077, 2015.

[5] Y. Li, M, Safari, R. Henderson, and H. Haas, "Optical OFDM with single photon avalanche diode,” Photonics Technology Letters, IEEE, vol.27, no. 9, pp. 943-946, 2015.

[6] Jeganathan, J., Ghrayeb, A., Szczecinski, L., Ceron, A., Space shift keying modulation for MIMO channels[J], IEEE Transactions on Wireless Communications, 2009, 8, 3692 - 3703.

[7] Renzo, M., Haas, H., Improving the performance of space shift keying (SSK) modulation via opportunistic power allocation[C], IEEE Communications Letters, 2010, 14, 500-502. 